\title{
RFID Components, Applications and System Integration with Healthcare Perspective
}

\author{
Kamran Ahsan \\ Staffordshire University \\ UK
}

\section{Introduction}

RFID (radio frequency identification) technology has already proved its use in various areas such as security, library, airline, military, animal forms, sports and other areas. RFID is being used for various applications in many industries. For example, equipment tracking, access controls including personal and vehicle, logistic, baggage, items security in departmental store. The main advantages RFID provide is resource optimization, quality customers' care, enhanced accuracy, efficient business processes, and effective business and healthcare processes. RFID can help is recognizing contextual knowledge and can help to improve objects predictability for certain processes. However, it is necessary to study RFID components for using these in healthcare environment. RFID main components are antennas, tags and readers. The investigation of these components provides an understanding of its use in healthcare settings and integration in healthcare processes.

This chapter studies the RFID components such as Antenna and reader. This chapter discusses the RFID active and passive tags, and compare these tags including advantages and disadvantages of RFID system. In this chapter, RFID applications are explored and technical model is analyzed. It also considers the healthcare perspectives and RFID use within healthcare settings. This study constructs a model for connected RFID applications which provides quick support for various healthcare functions and enhances flexibility for various systems' components integration.

\section{Motivation of RFID technology}

Existing research suggests that healthcare organisations are adopting information technology, specifically mobile technology throughout the world including the USA, Europe and UK (Bharadwaj et al., 2001). In the UK, the NHS (NHS-UK, 2009) is keen to adapt mobile technology for better information handling and this argument is supported in this chapter. However, real-time techniques and contextual knowledge management concepts for instant care is somehow neglected (Watson, 2006). Healthcare processes are volatile and the context of information changes rapidly. New technology has not considered information within their context. The context of information is more complex in healthcare in comparison to other industries. Although businesses have already started to develop and implement mobile technology for handling contextual information to improve processes but the same approaches cannot be adopted in the healthcare industry due to dominant 
knowledge use rather than just information and substantial human involvement (Connecting for health, 2009). However, the proven technology in business scenarios such as RFID can be adopted for a healthcare situation with the appropriate modelling of its use. Managing context for any information is a difficult task but information systems play an important role into it but contextual knowledge is even more difficult and need location, time and duration for information for providing context to any knowledge (Bharadwaj et al., 2001). If knowledge gets support with context of objects' location, duration and time then this contextual knowledge can improve various situations for resource optimization and instant better actions. RFID technology use is critical to get this knowledge and providing context to it. RFID can also support tacit knowledge on a real-time basis in healthcare situations such as patients moving between locations to get medical treatment and a change in their medical condition at the same time. The utilization of tacit knowledge is crucial but it needs context environmental knowledge for instance actions. One of the properties of RFID is to provide instant location information of any object associated to it and this can play a vital role for tacit knowledge support and managing other environmental knowledge. Advanced use of RFID technology can integrate patients' flow processes appropriately and support patients' treatment processes by deterministic patients' movement knowledge (location and time etc.) within hospital settings (Connecting for health, 2010).

In a healthcare situation the patients' movement processes are subject to change due to various reasons including a change in the patients' medical condition, due to the unavailability of a particular resource at any given time and the unpredictable duration of any medical procedure (DH-UK, 2009). When processes are executed according to a plan and schedule then it consumes healthcare resources in a predicted way, if processes change due to any of the reasons described above then time and resources may be misused or processes become unpredictable. These situations consume resources unnecessarily and the instability of one process at one location may affect other processes at another location. So, the use of RFID technology is crucial for determining situations through getting time and location of an object within healthcare settings. Use of RFID technology is important for better process management including improved decision-making.

\section{RFID utilisation}

RFID works for identification of items/objects (Bohn, 2008). Sometime it only identifies item category or type but it is capable of identify items/objects uniquely. RFID also enables data storage for remote items/objects through remotely access items information (Schwieren1 \& Vossen, 2009). RFID system consists of RFID tags, RF Antennas, RFID readers and back-end database for storing unique item's ID. In RFID systems, RFID tags use as unique identifier, these tags associate with any items, when system reads these unique tags then information associated with that tags can be retrieved. Antennas are first point of contact for tags reading. Reader can only work with software resides in reader's ROM (Glover \& Bhatt, 2006). RFID system is based upon tags and reader's communication and range of communication/reading depends on operating frequency. When antennas deduct tags then an application which is part of reader manipulates tags' information in readable format for the end user. There is a great amount of research being conducted to improve the efficiency of RFID systems, increasing the accuracy of RFID reader and the feasibility of RFID tags. Although RFID accuracy needs more enhancement and efficiency yet to be increase but still RFID system is used in many applications (Bohn, 2008). There are a variety of tags, readers 
and antennas types are available. Before implementing RFID system, selection among these types needs understanding of these types in relation to their feasibility, capabilities and reliability. It is also necessary to understand combinational use of these types for implementing a single feasible RFID system.

\section{Research approach}

Qualitative research methodology is followed for observing the patients' flow situation within hospital settings. It includes observation and open interviews. This study tries to find out the pattern within hospital condition, knowledge elements for healthcare processes and priority of each knowledge element for knowledge factor integration with the help of location deduction technology (RFID). Some individual scenarios are considered within patients' movement processes and understanding is build for integration of RFID integration within these processes. In this respect, qualitative methodology is sufficient for including each knowledge element and device a way of handling these elements through location deduction technology. This chapter explores RFID technology with its kinds, types and capabilities. It is conferred that how RFID technology can be generalised through generalise technical model. It is discussed that how component layering approach can be feasible for integrating various healthcare management disciples for providing improved management. Healthcare knowledge factors are considered for supporting knowledge elements through RFID technology to improve healthcare situation.

\section{RFID evaluation}

RFID technology continues to evolve in past years in terms of various shapes of tags for increase its feasibility of its use, fast reading rate of reader and range of antennas etc. The use of RFID also evolves due to enhancement in its components. As the accuracy increases, the use of technology also increases such as baggage handling, goods delivery tracking and courier services. RFID system enhancement also evolves automation applications development e.g. automatic toll payments, automatic equipment tracking and document management etc. (Garfinkel \& Rosenberg, 2005). In this connection, the evolution process of RFID with respect to past few decades can be seen in figure 1 .

\section{How RFID system works}

The basic unit of RFID system is tags and tags have its own unique identification number system by which it recognizes uniquely. These unique identification numbers save in tags' internal memory and it is not changeable (read-only). However, tags can have other memory which can be either read-only or rewrite able (Application Notes CAENRFID, 2008). Tag memory may also contain other read-only information about that tag such manufactured date. RFID reader generates magnetic fields through antennas for getting acknowledgement from tags (Garfinkel \& Rosenberg, 2005). The reader generates query (trigger) through electromagnetic high-frequency signals (this frequency could be up to 50 times/second) to establish communication for tags (Srivastava, 2005). This signal field might get large number of tags data which is a significant problem for handling bulk of data together. However, this problem can be overcome through filtering these data. Actually software performs this filtering and information system is used to supply this data to data 


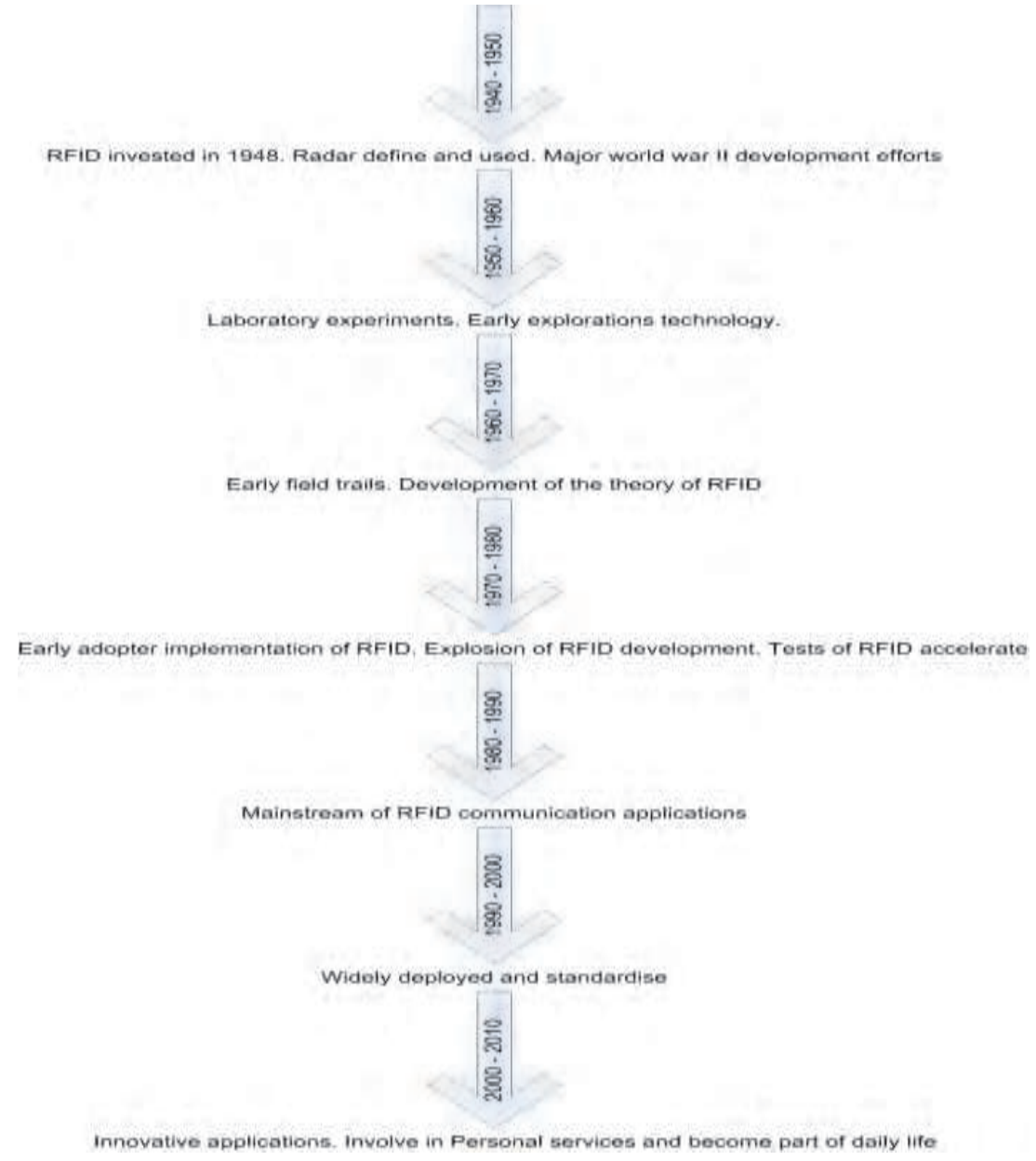

Fig. 1. RFID evolution: over past the few decades (Srivastava, 2005)

repository or use any other software procedures to control data according to the need and system capability (Srivastava, 2005; Application Notes CAENRFID, 2008). This piece of software works as a middle layer between user application and reader because the reader normally does not have the capability to handle bulk data at once; it has the job to supply reading data to user application for further process (Frank et al., 2006). This buffering capability may supply data from reader to information system interface (user interface) directly or may provide and use some routine to save into database for later exploit, it is depend on user requirement.

Reader and tags communication can be maintained through several protocols. When the reader is switched on then these protocols start the identification process for reading the tags, these important protocols are ISO 15693, ISO 18000-3, ISO 18000-6 and EPC. ISO 15693 and ISO 18000-3 protocols are used for high frequency (HF) and, ISO 18000-6 and EPC protocols are used for ultra high frequency (UHF). Frequency bands have been defined for 
these protocols and they work within specified range such as $\mathrm{HF}$ has $13.56 \mathrm{MHz}$ and UHF between 860 - $915 \mathrm{MHz}$ (Application Notes CAENRFID, 2008). Reader modulates tags responses within frequency field (Parks et al., 2009).

The reader handles multiple tags reading at once through signal collision detection technique (Srivastava, 2005). This signal collision detection technique uses anti-collision algorithm, the use of this algorithm enables multiple tag handling. However, multiple tags handling depend on frequency range and protocol use in conjunction with tag type which can enable up to 200 tags reading at single time. Reader protocol is not only use for reading the tag but also perform writing on to tags (Application Notes CAENRFID, 2008).

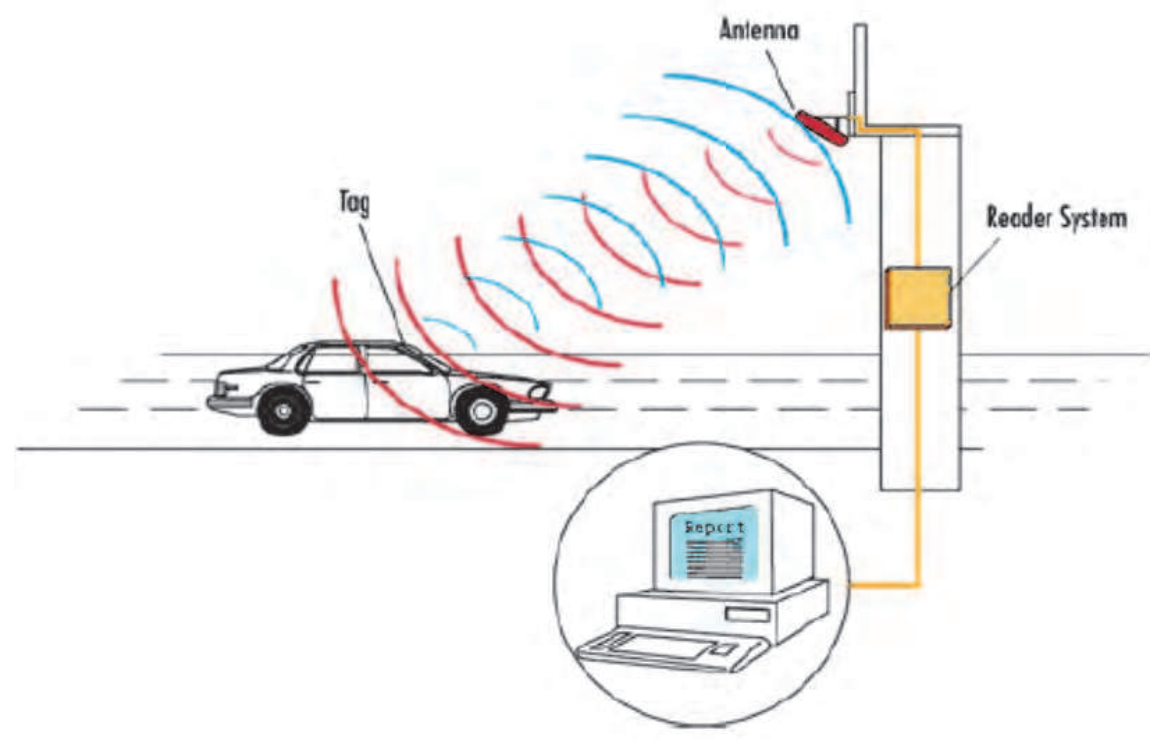

Fig. 2. A typical RFID system (Application Notes CAENRFID, 2008)

The use of the reader within RFIFD system can be seen in figure 2. This figure also define the overall cycle of tag reading by reader through antenna and transforming data into communicate able form to user applications.

\section{How RFID system works}

RFID system deducts tags within antennas' range and performs various operations onto each tag. The RFID system can only work effectively if all RFID components logically connect together and these components need to be compatible with each other. Thats' why understanding of these separate components is necessary. Implementation of complete RFID solution is only possible through integration of these components which needs understanding of compatibility for each component, realisation of each components compatibility needs property study for these components (Sandip, 2005). These components are gathered and defined as under. Also integration of these components can be understood with figure 3. 
- Tag has unique ID and use for unique identification; tags are attached with objects in RFID solutions.

- Antenna use for reading tags; antenna has its own magnetic field and antenna can only read tags within these magnetic fields.

- Reader works for handling antenna signals and manipulate tags' information.

- Communication infrastructure use for reader to communicate with IT infrastructure and work as middle layer between application software and reader.

- Application software is a computer base software which enable user to see RFID information, this can be database, application routines or user interface.

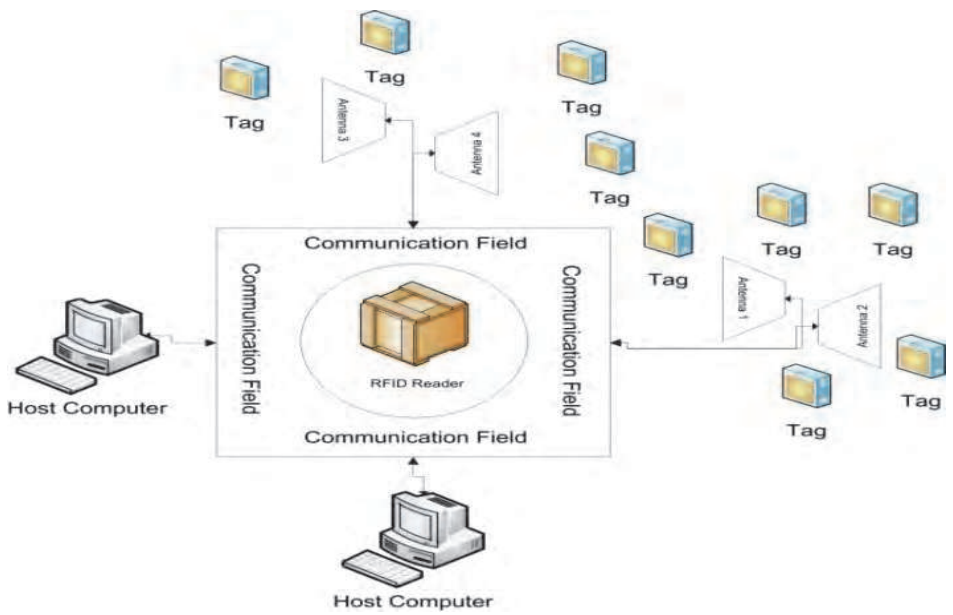

Fig. 3. Components of an RFID system

\section{RFID tags}

RFID tag has memory in the form of a microchip which store unique code for tag's identification, this unique identification called tag's ID (Application Notes CAENRFID, 2008). The microchip is a small silicon chip with embedded circuit. Numbering technique is used for providing unique identification (Garfinkel \& Rosenberg, 2005). This microchip could have read-only or writeable characteristics depending on tag type and its application within RFID solution. These characteristics depend on the microchip circuitry which has form and initialize during tag manufacturing (Miller \& Bureau, 2009). Some tags (read-only) re-programming is possible but need separate electronic equipment for re-programming read-only tag's memory. Writable tags also know as re-write tags do not need any separate equipment and reader can write data on it, depend on the protocol support, if reader have writing command capability and tags are in range. Tags selection is very important for feasible use in RFID solution. This selection is dependent on the tag size, shape and material. Tags can be integrated in varity of material depending on the need of the environment. The tag is embedded in plastic label in form of a microchip, stick able material for documents handling, plastic material with use of pin for use in cloths material are the good examples to be consider (Frank et al., 2006). Various forms of tags with respect to its sizes and shapes can understand with figure 4 . 


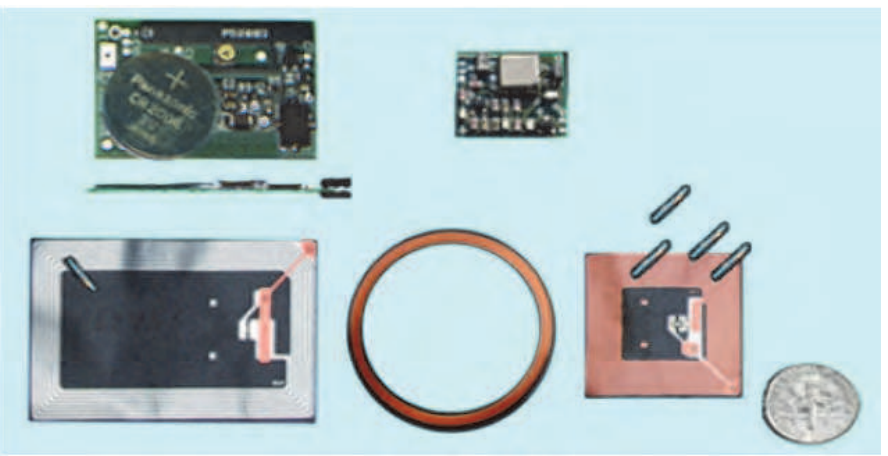

Fig. 4. Varity of RFID tags (various shape \& sizes) (Frank et al., 2006)

Classification of RFID tags is also possible with respect to their capabilities such as readonly, re-write and further data recoding. Further data recording examples are temperature, motion and pressure etc. (Narayanan et al., 2005). Compiled tags classification into five classes previously gathered by Narayanan et al. (2005) is shown in figure 5.

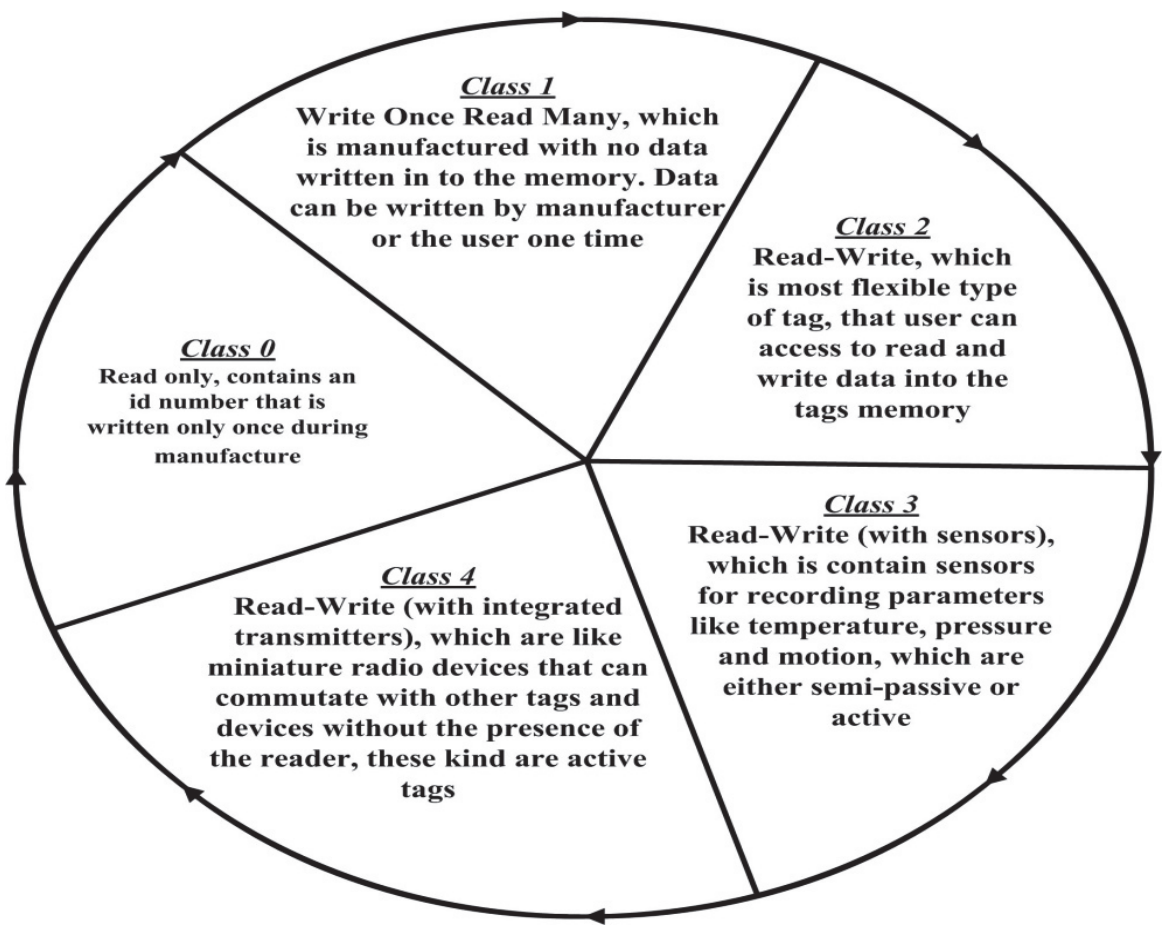

Fig. 5. RFID tags classifications (Narayanan et al., 2005)

Active, semi-active and passive are the three main tags types. Tags made up with few characteristics which may vary slightly depending on type of tag, due to which their use can be change in RFID solution (Zeisel \& Sabella, 2006). So, selection of tags depends on the 
functional need of RFID application. The main difference is between active and passive tags because semi-active tags have mix of both tag's characteristics (Application Notes CAENRFID, 2008). These types differentiate upon memory, range, security, types of data it can record, frequency and other characteristics. The combinations of these characteristics effects tags' performance and change its support and usefulness for RFID system (Intermec, 2009). The main tag types (active and passive tags) are compared in following figure 6.

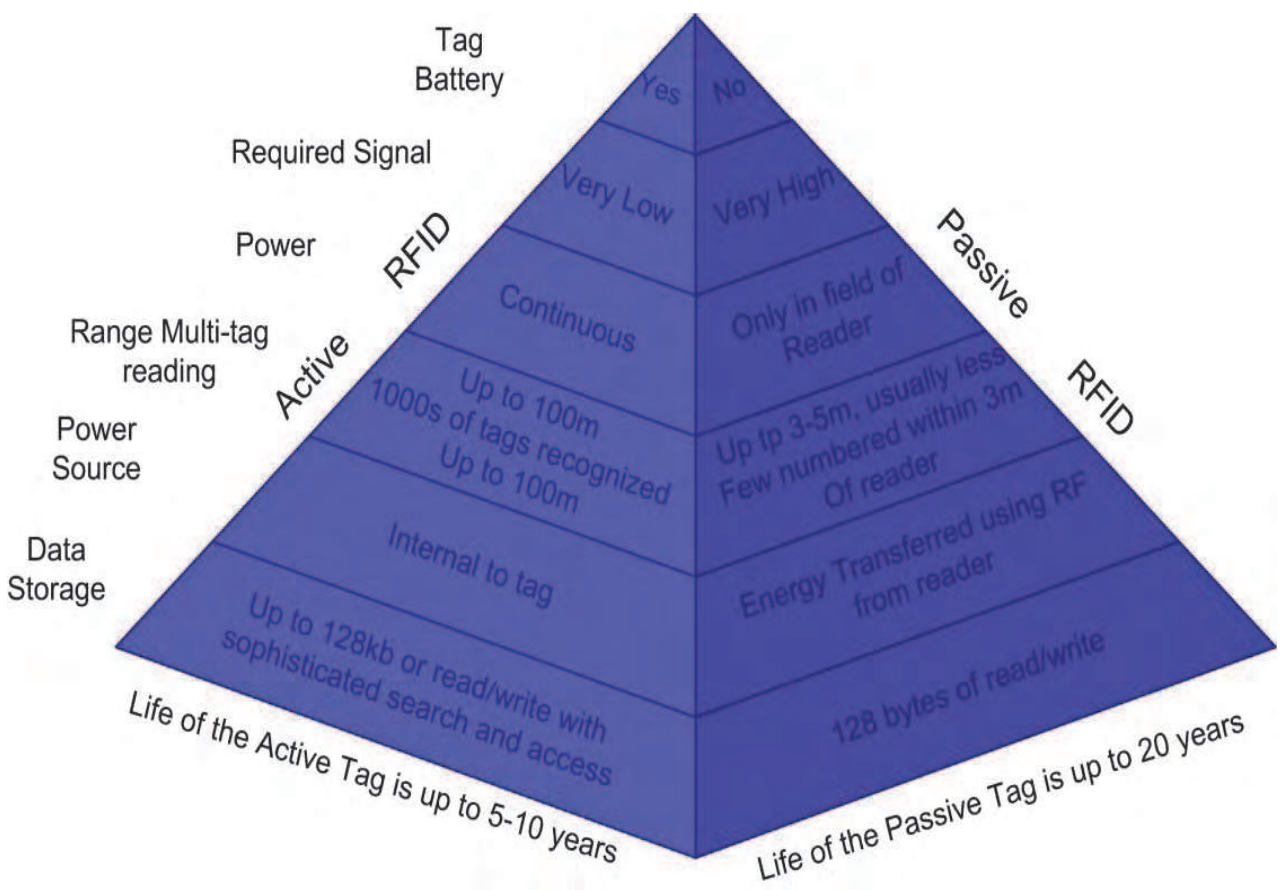

Fig. 6. RFID active and passive tags comparison

\subsection{Tags physical features}

The tags have various physical features such as shape, size and weight. Consideration of these features depends on environment tag being used. Classified tag's physical features are as under.

- Smart labels can embed in layers type materials such as papers.

- Small tags can embed objects other then flat panel such as clothes and keys.

- Plastic disks can use for attaching with durable objects and use in tough environments such as pallets tagging use in open air.

\subsection{Tags capabilities}

The tags can also be differentiated with respect to tags capabilities and performance (Schwieren1 \& Vossen, 2009; Garfinkel \& Rosenberg, 2005). Following is the list for tags capabilities. 
- Anti-collision capability of a tag, tags having anti-collision can enable reader to recognize its beginning and ending which help reader to read all tags in its range.

- How tags get its power source such as active has its own battery and passive get power from reader through its magnetic field.

- Conditions of tag environment such as use in water.

- Tags data writing capabilities such as write one time or many times onto tag memory.

- Coupling mechanism tag use such as magnetic, inductive, capacitive and backscatter. Coupling mechanism determines tags information and power sharing methods.

- If tag can work for more than one protocol which enable tags to work with different types of readers.

- Tags with encrypted data handling feature.

- Either tag has two way communication (full duplex) or one way communication (half duplex).

\subsection{Tags standards}

Understanding of tags standards is necessary for working with various systems, protocols and procedures. It is dependent on organisational policies and scope of RFID system. Tags standards enable interoperability capability to RFID solutions (Sandip, 2005). For example, if tags have standardization and its uniqueness can be identified across different systems then it enhances the use of standard tags (Schwieren1 \& Vossen, 2009). The spectrum of tags can be single situation such as tags use in single warehouse, multiple spectrums such as same tags use in logistic and supply chain and need recognition across different organisations and various systems (Shepard, 2005). The selection of tags standards within RFID solutions depend on these spectrum. Following three standards are gathered by (Shepard, 2005).

ISO/IEC 18000 tags: This standard works for various frequency ranges including long range (UHF), high frequency (HF), low frequency (LF), and microwave. This standard supports various principle and tags architectures. The range of tag identification includes 18000-(1 to 7).

ISO 15693: In this standard tag IDs are not as unique as ISO 18000. Although vendors try to build unique tags with certain specification and coding but it is not globally unique. These standard tags most often use in smart cards for contact-less mechanism. However, it is also use in other application but in local scenario (not global) e.g. supply chain and asset tracking etc.

EPC tags: It is the standard for maintaining the uniqueness under certain management bodies. It carries out tags uniqueness with all the vendors associated with one management entity. Management entities carry their own EPC number technique and own the certain object class.

\subsection{Tags states}

Tags process recognize with its state within RFID working environment. Tags cannot have multiple states simultaneously. The set of tag states depend on the type of tag. However, these states generally include open state, reply state, ready state, acknowledge state, arbitrate state, killed state and secured state (Shepard, 2005).

\subsection{Tags frequencies and range}

RFID tags capability and working feasibility change according to its frequency and range. Tags prices and its use also vary in relation with tags frequency and range. Various frequencies and its range (working distance) can be seen in following figure 7 . 


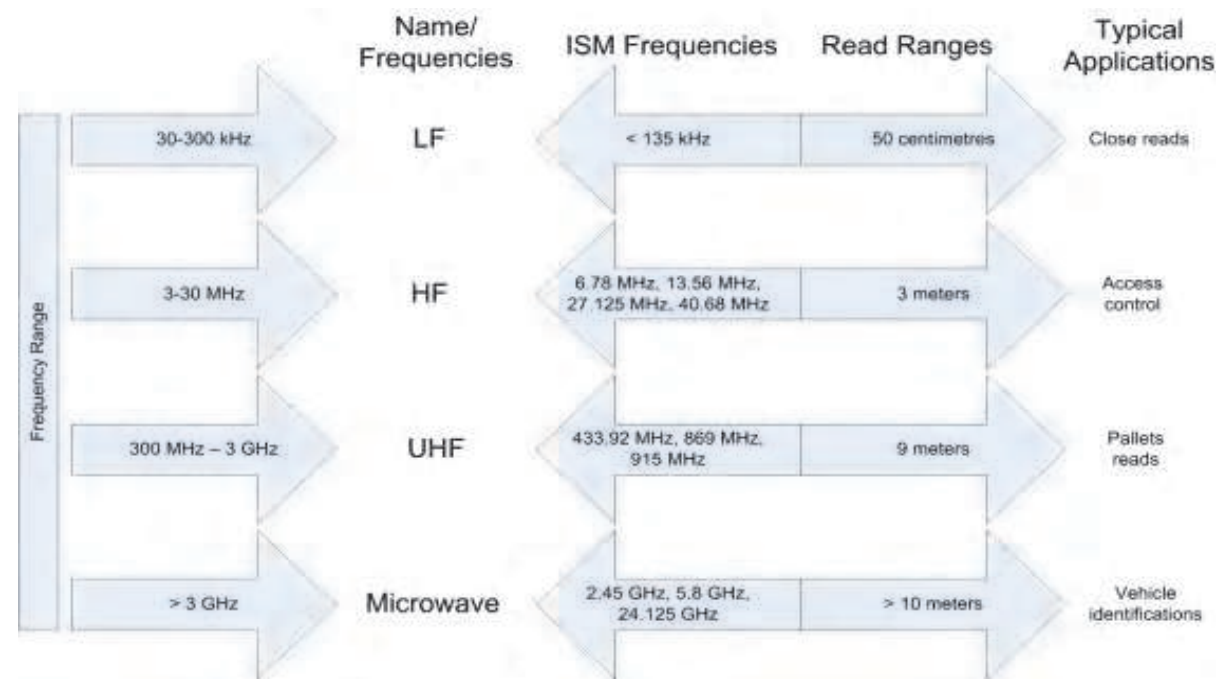

Fig. 7. RFID frequencies and ranges

The performance, range and interference feasibility depend on the frequency at which tags' operate (Zeisel \& Sabella, 2006). Different tags standard uses different frequency bands in which ISO and EPCglobal standard are major organisations working for UHF bands for developing international standards (Narayanan et al., 2005). However, full compatibility is still not achieved that's why most of the organisation obligated to use International Telecommunication Union principles (DHS, 2006). These principles include following frequency bands.

- High frequency can work up to one meter. It can embed with thin objects such as papers, that's why it is mostly use in sales points and for access controls. $13.56 \mathrm{MHz}$ is the frequency at which it work and it is less expensive to implement (Srivastava, 2005; Application Notes CAENRFID, 2008).

- Low frequency fulfils short range applications' needs. It is not effective for metal or wet surfaces and only works half of the high frequency range (maximum half a meter) (Frank et al., 2006). Low frequency works on $125 \mathrm{KHz}$ (Application Notes CAENRFID, 2008).

- Ultra high frequency has better read rate and large number of UHF tags can be recognize at one time. It has also good better read range and three times with high frequency, it is capable to read tags up to three meters. However, range can be reduced in wet environment. It works between $860-930 \mathrm{MHz}$ frequencies (Srivastava, 2005).

- Microwave has less read range and it works within one meter. But it has rate of reading is faster than UHF with very little affect on wet and metal surfaces. It works on Giga Hertz frequency and faster than LF, HF and UHF, that's why it can work better for vehicle access application (Application Notes CAENRFID, 2008).

\subsection{Tags fields}

Active tags have its own power but passive tags get the power from antenna based on readers' signal to antenna (Application Notes CAENRFID, 2008). Passive tags response or communication signal is based on the power it gets from antenna. Following two methods passive tags use for getting power from reader. 
Far field uses coupling methods with the electric signals within field of antenna as shown in figure 8 . These tags embed their signal in reverse order with antenna signal using some standard format so that reader can recognize the tag signals (Frank et al., 2006).

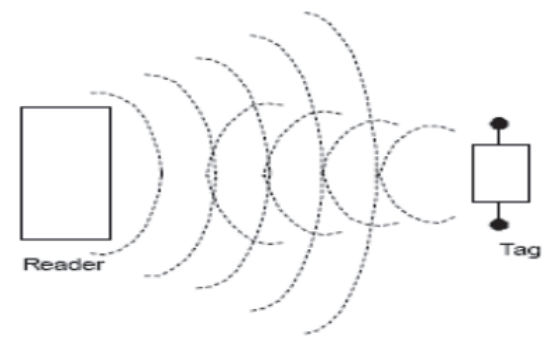

Fig. 8. RFID far field methodology (Application Notes CAENRFID, 2008)

Near field uses inductive coupling within magnetic field of an antenna as shown in figure 9 (Application Notes CAENRFID, 2008).

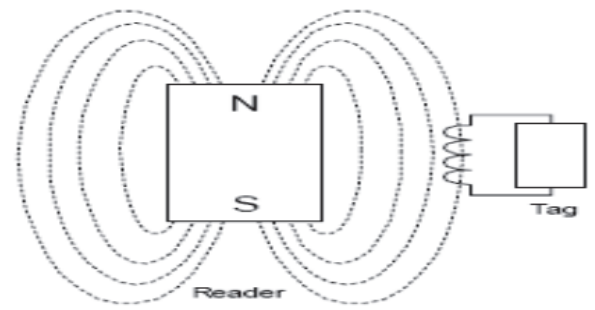

Fig. 9. RFID near field methodology (Application Notes CAENRFID, 2008)

These methods are use in different kind of applications and system is based on different circuitry (Meiller \& Bureau, 2009). Far field is appropriate for microwave and UHF because it can work in longer range and near field is suitable for LF and HF because it can only work within shorter range (Meiller \& Bureau, 2009; Parks et al., 2009).

\section{RFID antennas}

RFID antenna is the middle-ware technology or component, it work between reader and tag and provide energy to tags in some cases (passive tags). It performs tags data collection. It shapes can be altered depend on the application and feasibility of use but shapes varies the range of antenna.

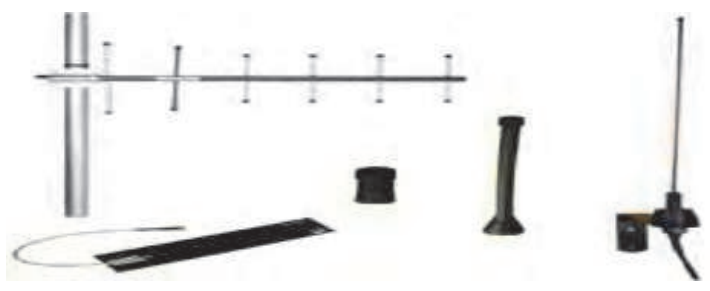

Fig. 10. RFID antennas types (Intermer, 2009) 
Antenna has various shapes and some of them can be seen in figure 10. Antennas can be differentiated with various properties such as direction of signals (tags reading direction) and polarities. Stick antennas, gate antennas, patch antennas, circular polarized, di-pole or multi-pole antennas, linear polarized, beam-forming or phased-array element antennas, Omni directional antennas and adaptive antennas are the types of antenna commonly use in various applications (Zeisel \& Sabella, 2006).

\section{RFID readers}

RFID reader is a external powered equipment used in RFID system for producing and accepting radio signals (GAO, 2005). A single reader can operate on multiple frequencies and this functionality depends on the vendor (Application Notes CAENRFID, 2008; Frank et al., 2006), it can have anti-collision algorithm/procedures for deducting multiple tags at one time. RFID reader works as middle-ware between tag and user application. Reader is the central part of the RFID system and communicates with tags and computer program, it supply tags information to a computer program after reading each tags unique ID. It can also perform writing onto tag, if the tag is supported. Although the reader can have multiple frequency capability but it works on a single frequency at a time. The reader can communicate with the computer program and need either wired or wireless connection with the computer. This reader can use a wire connection with any of the following: USB, RS-232, and RS485. Otherwise, the reader can connect with the computer through Wi-Fi (known as network reader) (Sandip, 2005; Zeisel \& Sabella, 2006). The reader provides various management techniques and functionality to computer programs (Zeisel \& Sabella, 2006) through various built-in functions/components, these components can be understood with following figure 11.

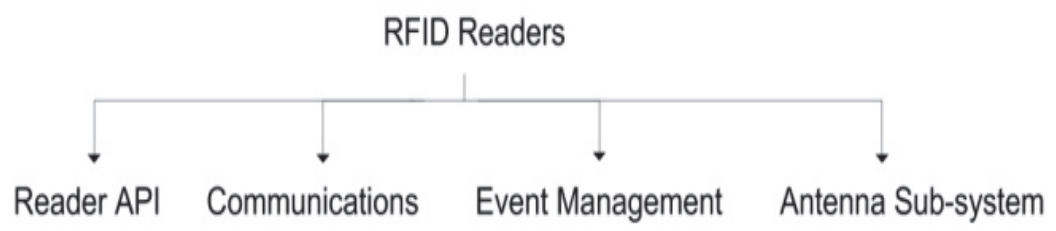

Fig. 11. RFID reader logical components

\subsection{Reader protocols}

Although vendors are trying to implement reader with common protocols but the standardization of RFID readers' protocol is not achieved yet which is why readers are not interoperable (Glover \& Bhatt, 2006). An organisation cannot replace a reader easily after RFID solution implementation. However, there are some common capabilities RFID readers provide. Command, sensor, observation, alert, transport, host and trigger are the most common capabilities provide by RFID readers.

Synchronous and asynchronous are two types of communications used by readers (Shepard, 2005). In synchronous readers' communication with host, the host requests the update with the reader (Garfinkel \& Rosenberg, 2005). In response to that, the reader sends the list of updates to the host. In case of asynchronous communication, the reader sends notification to the host about its observation. This notification can be sent to host upon request or immediately after new observations, it is dependent on the requirement and trigger 
mechanism of RFID system (Shepard, 2005). Both types of communication can be understood from the figure above 12 .

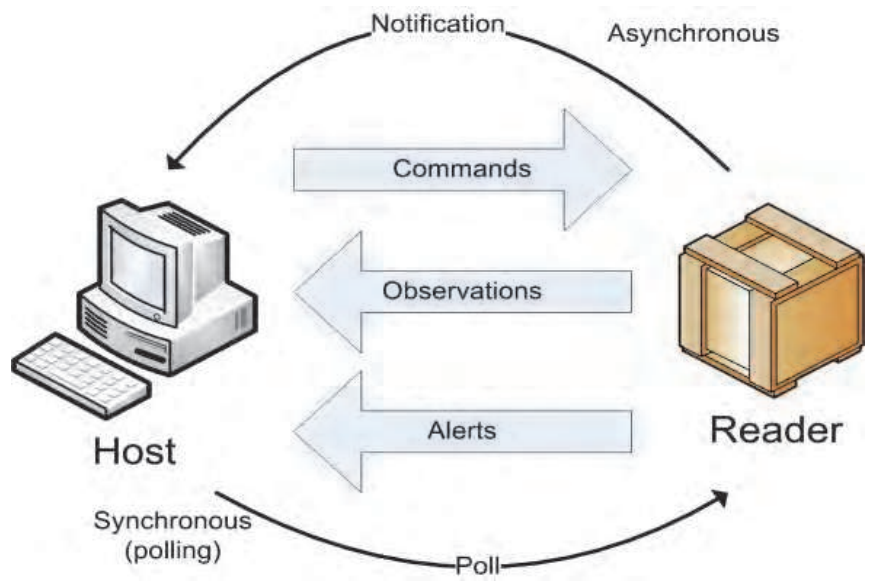

Fig. 12. Information flow and a/synchronous communications (Shepard, 2005)

In both of these communication methods the information flow has three types which include; observation, host pass commands to reader and reader pass alerts to host (Shepard, 2005).

EPCglobal is the most common and most accepted protocol. EPCglobal provides three layers for communications; these layers are message, transport and reader (Zeisel \& Sabella, 2006). The messaging layer use transport layer to pass messages according to the format defined by the reader layer (Garfinkel \& Rosenberg, 2005). Connection commands, host commands, security and reader notifications are the most common command deal by message layer. Reader layer identifies the format of the message transport between host and reader. The transport layer is responsible for network support and establishes communication between reader hardware and computer operating system (Zeisel \& Sabella, 2006).

\subsection{Reader interfaces}

RFID reader communicates with the computer program by using the reader's protocol as described in the previous section. The reader should be capable to handle various types of commands which include management of events, communicate with applications and adapter. These also provide various kinds of interface with the reader. Figure 13 shows the three kinds of interfaces most commonly any reader provide.

The reader provides a command set for communicating with user interface of computer programs. These command set understands the reader properties and provides functionality for using a particular reader (GAO, 2005). These command sets are known as application program interface (APIs) (Frank et al., 2006). If organisation builds their application program based on a specific reader then this computer program needs to use APIs provided by particular reader (Application Notes CAENRFID, 2008). Customize application might not be compatible with other reader but in this case a vendor upgrades their readers hardware, organisation might be able to use those readers. Vendors most often provide the compatibility of previous APIs in the case of an upgrade (Shepard, 2005; Frank et al., 2006). 
In that case, organisations can upgrade their hardware using the same application but organisations must refer to vendors' device specifications before any upgrade. Some vendors also provide application compatibility with a range of their hardware through consistent APIs set (Zeisel \& Sabella, 2006). This mechanism provides adaptability of various readers with same application for some extent. Reader interface within RFID reader provides the filtering for the reader data (raw data) before sending to application program. Reader provides the raw data to reader interface, this data could be bulk (depend on the environment), reader interface need to find relevant data within bulk data provided by reader. This functionality reduces the overhead of program interface or application program and as well as provide low traffic for communication between computer software and reader due to sending only relevant data.

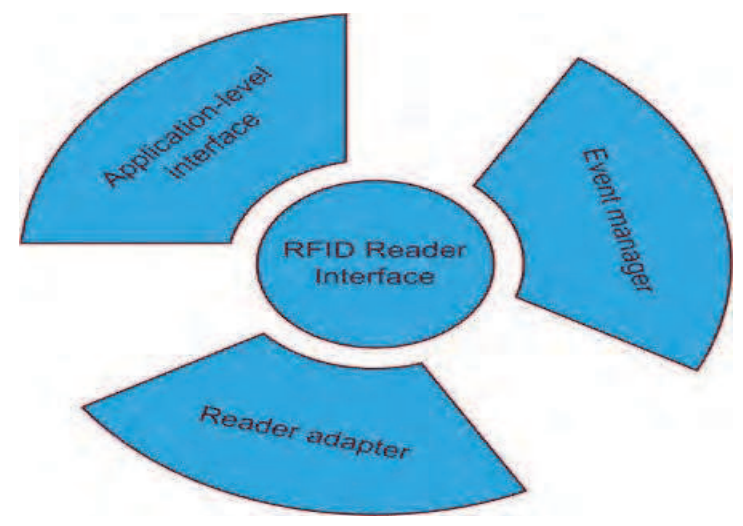

Fig. 13. Reader interface (Frank et al., 2006)

\section{Advantages and disadvantages of RFID systems}

The use of RFID solutions have been recognize by many industry. However, the appropriate level of RFID components combination and selection of these components according the suitability of organisational situation and environment can make it beneficial. Otherwise, RFID system with the inappropriate combination and selection of RFID components may generates error or does not work effectively which could be increase organisational operational cost and may affect customers' good will. The list of advantages and disadvantages can be seen in table 1 (Meiller \& Bureau, 2009).

\begin{tabular}{|c|c|}
\hline Advantage & Disadvantage \\
\hline High speed & Interference \\
\hline Multipurpose and many format & High cost \\
\hline Reduce man-power & $\begin{array}{c}\text { Some materials may create signal } \\
\text { problem }\end{array}$ \\
\hline High accuracy & Overloaded reading (fail to read) \\
\hline Complex duplication & \\
\hline Multiple reading (tags) & \\
\hline
\end{tabular}

Table 1. Advantages and disadvantages of RFID system 


\section{RFID general technical model}

So far it has been studied that RFID system varies with respect to various features. These features include physical features, components, standards, capabilities, frequencies, states, ranges, protocol, interfaces and readers. Due to variable RFID features and compatibility issues, it is very difficult to develop integrated RFID solution (Glover \& Bhatt, 2006; Application Notes CAENRFID, 2008). If organisation tries to build RFID solution with future compatible hardware then it makes RFID components' selection, implementation and integration even more complex. However, RFID regulatory bodies try to provide safe and less conflict (radio and other frequency using equipment) RFID standards and vendors try to provide interoperable equipments. But true interoperability is not possible until globally accepted standard not developed and manufacture adapt single standard or at least limited standards. In this context, two main organisations are doing efforts for providing globally accepted standards (Application Notes CAENRFID, 2008). These organisations (EPCglobal and ISO) are trying to develop unique standard for RFID tags so that tags can be used in wide spectrum throughout the world including supply chain and transportation. However, there still no standard is available for compete RFID system or solution. In this connection, it is necessary to understand RFID tags, air interface, reader, reader's programs including data protocol processor and physical interrogator, needs of application programs, and application commands and responses in integrated way. For this purpose, a generalise model for RFID system is provided for better understanding (see figure 14).

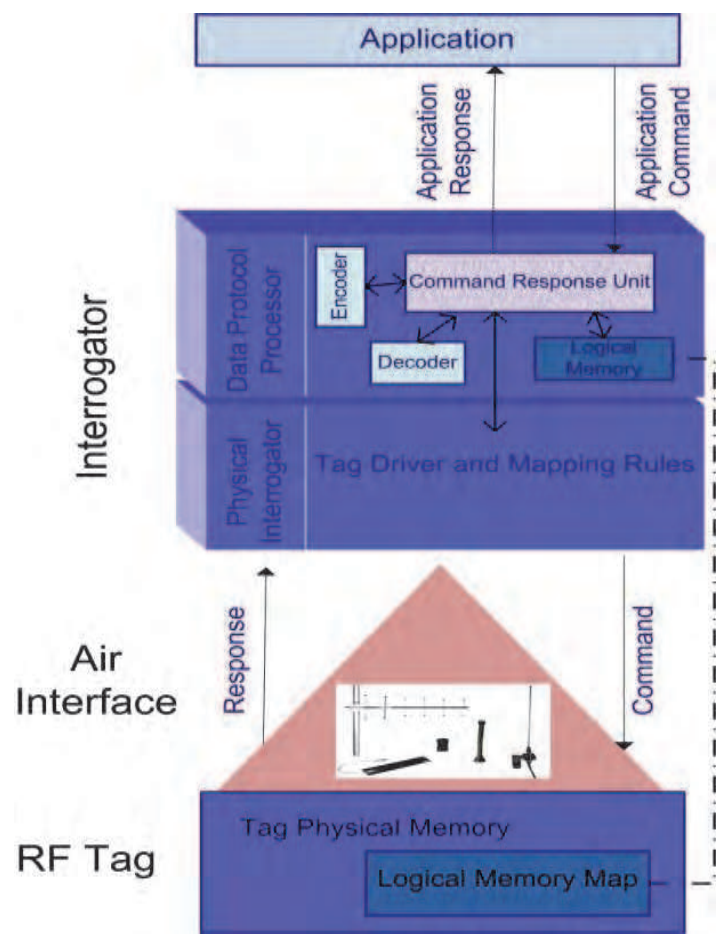

Fig. 14. RFID general technical model 


\section{RFID applications}

Before understanding the use of RFID system for contextual knowledge management, it is appropriate to study the use of RFID applications for other scenario or situations with respect to short and long range RFID applications. For this purpose a generalised RFID application model has been presented as shown in figure 15.

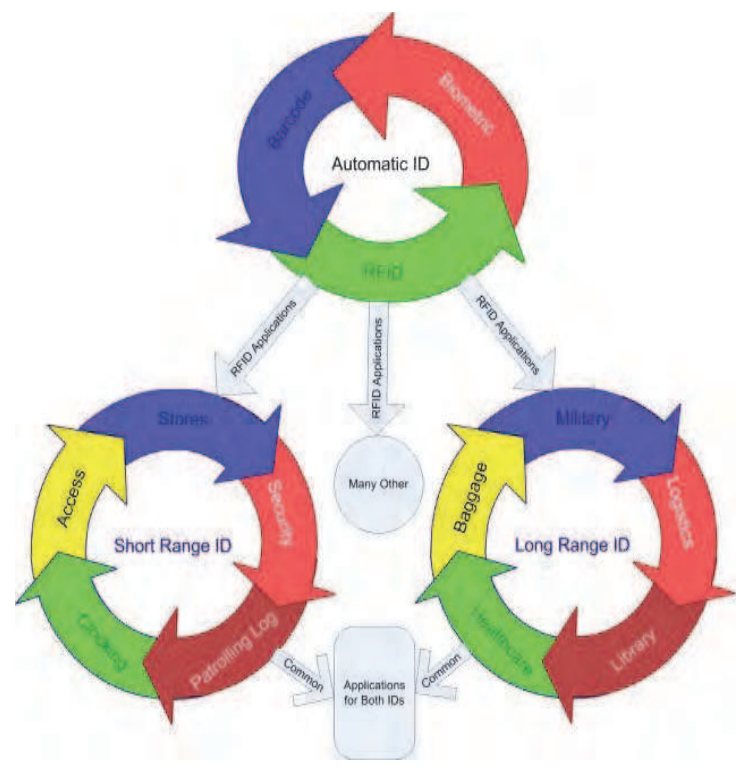

Fig. 15. General RFID applications according to its capabilities

From a RFID application perspective, RFID has two categories: short range and long range. In short range application tag need to show to reader. Application works perfectly in short range if object or tag access the reader one by one. For example, in access control, only employee or specified person can access the secure building and building has several divisions each division has particular set of authorized people, who can access certain divisions. Person need to show the tag near the reader before every secure door for enabling the system to decide whether door should open for that person or not. In long range applications, tags do not need reader near, as compare to short range. For example, tags place on every book in the library and user can easily access the exact book shelf for the required book. This enable automated inventory control. Readers can read the multiple items simultaneously from the distance. Reading distance can vary depending on the frequency and type of reader. This section further discusses the most common use within short and long range applications.

\subsection{Security and control applications}

RFID system can be used for control access and security; it is also useful for audit purposes. These applications are not only granted permission to access a particular secure zone but also record who is accessing, from which location/areas, at what time and for how long duration. These types of RFID systems can maintain building and departmental security 
including privacy. This type of RFID solution is also workable for equipments and objects controls (Shepard, 2005).

\subsection{Patrolling log applications}

In this application, security firms use RFID system to control their security guards and use RFID data for various purposes. These purposes include performance checks, data can be used in reference to unexpected event and audits for a secure area etc. The main difference in this type of application with other applications is that the reader is variable and the tag is fixed, i.e. the reader go near to the tag rather tag swap near to the reader. In security patrolling several numbers of tags can be fixed throughout the building and the security guard needs to swap the reader with each tag (checkpoint) in a sequential order within an allocated time and repeat this process throughout his/her shift. The reader records each swap which can be transferable to a computer program later on for audit or other purposes.

\subsection{Baggage applications}

Baggage handling and package delivery is a complex task and needs a large number of human involvement which is an expensive resource. Humans do various operations from receiving packages, sorting, assembling and distribution. Due to human involvement the error rate can be high. While the use of RFID tagging system not only reduces human involvement but also automates the process for certain extend which enable fast packages delivery. RFID solutions for baggage and packaging firm including airline industry are better for their effective operation and it reduce the complexity for overall system.

\subsection{Toll road applications}

RFID can provide the automated toll collection and maintain the traffic flow without stopping vehicles for payment. In these type applications, vehicles either pre-pay their toll yearly quarterly or monthly or other kind of scheme can be applicable such as pay-as-you go. In any case, the reader can recognize and record the vehicle entry at each toll which can be calculated later on by an application program. These applications not only help in toll collection and maintain traffic flow but also provide statistical data for any road which can be utilized for analysis and improvements (Shepard, 2005).

\section{Healthcare RFID applications}

RFID usefulness already proved its importance in security control, patrolling logs, baggage and packaging, and toll collections. Similarly, RFID can improve healthcare processes in various ways such as equipment handling, drugs transportation, blood samples administration, patients' notes management and others. RFID provide timely information about the objects associated with RFID solution, this feature enables better and up-to-date information about the processes link with these objects. The better information about objects around healthcare processes can further reduce the number of errors.

Information systems have many limitations, including failing to successfully update information automatically in relation to the location of an object, while RFID system can overcome this limitation through RFID advanced features. These RFID feature can save precious healthcare resources which can be utilized for patients' care such as a reduction in consultants' time for managing patients can enable consultant to give more time for 
patients' direct care. Healthcare processes involved with both processes patients' care and non-patients' care. Patients' care includes; fever test, blood pressure and scan etc. which is direct care. Non-patients' care includes notes management, movement of patients from one place to another, equipment handling from one ward to another ward, sending blood samples to pathology, preparing management reports and managing beds etc. These nonpatients' care processes are known as indirect care. If indirect care processes improve within healthcare settings then it saves healthcare practitioners' time which can be utilized for direct care. RFID systems can also provide the automation to some extent, this not only provide better planning but also enables real-time management. Most of RFID solutions focus on one issue such as management of objects; this management can be implemented for various things such as documents and equipments. If RFID solution only consider better management for healthcare then it might not be effective in various situations such as RFID solution for notes scanning by using low or high frequency tags. If RFID solution for notes scanning are implemented through low or high frequency then the RFID system may need physical scanning for each move like sales point scanning. In this case, healthcare practitioner intervention is required for each transaction. For example, if notes have to move from ward to theatre, it needs scanning at the ward for a system update and the theatre might need to scan again to enable the system to update the new location of the notes. When time is scarce this process may not occur effectively and the scanning chain may be broken at any time. In most of the cases, healthcare processes need automation to minimize the overhead from healthcare practitioners.

Implementation of RFID system for one modality or discipline can solve issues related to one modality or discipline. In case various RFID systems need implementation for different disciplines within healthcare, then these should be synergetic with each other. Otherwise it can increase the overhead for using more than one interface and systems for dealing with various objects and disciplines. RFID solutions can help direct care staff/practitioners to improve care but also effective for other staff such as radiologist and porters to play better role for overall process improvement. The use of RFID systems can be different for various stakeholders and each stakeholder can see different sets of information but it needs integration for consistent information.

The complexity of healthcare processes need appropriate level of RFID integration for several management disciplines. For example, better managing of notes can enable ward staff to handle patients in timely manner but if the equipment is not available staff still cannot execute patients' treatment procedures until right equipment is available. So, any procedure needs all process elements to handle patients' treatment efficiently. RFID can maintain elements availability for healthcare processes. However, it needs the study for RFID solutions in the context of process automation rather another overhead for healthcare practitioners such as automatic scanning when notes are presented in any unit within hospital settings rather than physical scanning of each set of notes. It is also necessary to investigate the integration level required for healthcare processes and classify each management disciplines for integration RFID application. In this connection, a model for possible management discipline within healthcare setting need RFID application in integrated way is presented (see figure 16).

It is very complex to provide a generic system for RFID application for every hospital setting due to a variation in processes. However, it appropriate to provide a generic model for adapting RFID technology solution in hospital setting which enable healthcare management 
to visualize healthcare processes in holistic way. Figure 16 is not the complete list for every healthcare management discipline and RFID technology can be used for other purposes within healthcare environment. However, this list provides the most urgent, appropriate and related management discipline which need integration through RFID application for overall processes automation. This section also elaborates few scenarios for further understanding of these separate but synergetic management disciplines. It is important to describe few scenarios in relation with RFID usability, it helps to understand generalized RFID application model for healthcare and it capability to improve healthcare processes within specific scenarios.

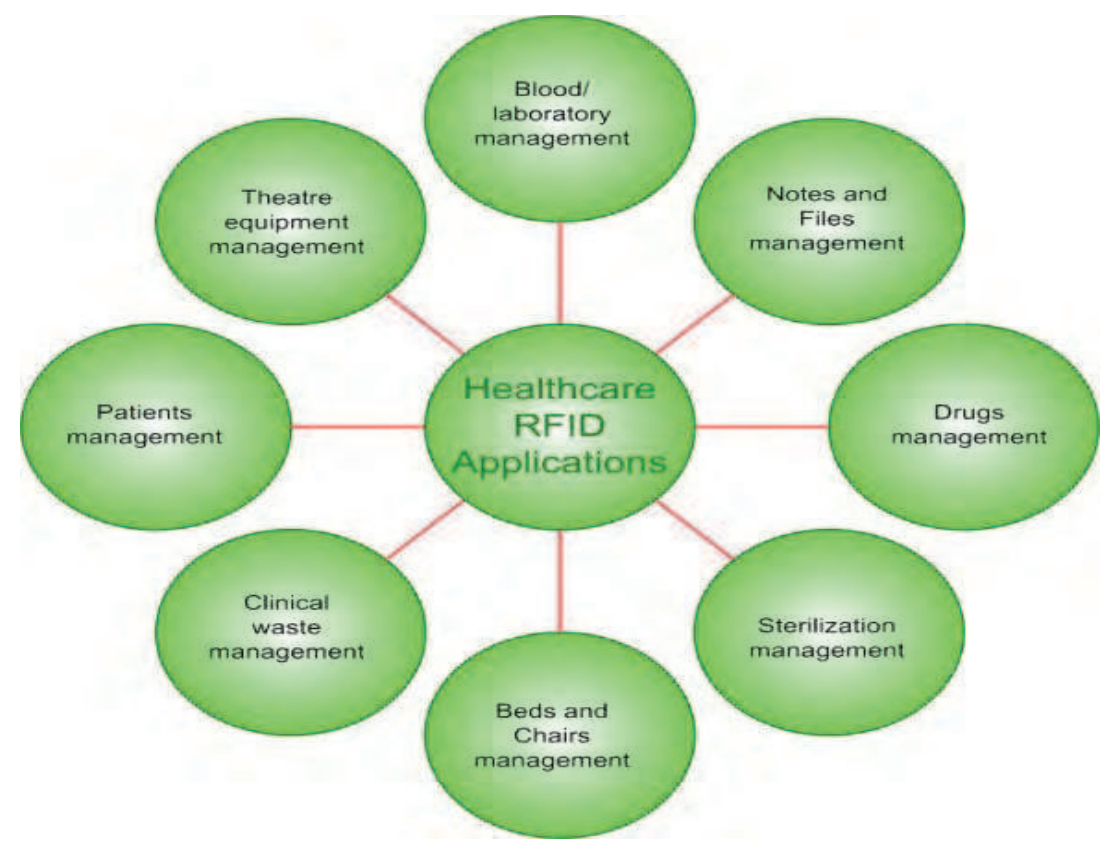

Fig. 16. Healthcare RFID applications

\subsection{Theatre inventory management}

Theatre inventory management is a separate healthcare management discipline which facilitates surgery and support theatres' other processes. Theatre equipment management is necessary because without equipment availability most surgeries are not possible such as hip replacement surgery needing the exact size and shape bowl for patient's surgery. If the exact surgery equipment is not available at the time of surgery then the surgery has to be cancelled. The theatre inventory management is complex due to various conditions. For examples, for some types of equipment the expiry date is very short and the theatre cannot keep this type of equipment for long periods of time that is why management like to keep inventory at low level for these types of equipments in order to avoid loss. Surgeons want to use new equipments in some cases depending on the patients' age and condition. Maintaining the level of inventory for these kind of equipments are very difficult. RFID can provide automation for the inventory update and support management to maintain their 
critical inventory effectively within low budget and meet the theatre requirement at the same time.

\subsection{Equipment sterilization management}

Sterilisation equipment control and maintenance is very complex due to a variety of functions. Sterilisation unit works in conjunction with theatre. Sterilisation unit deals with two type of equipment: consumable and reusable. Both types of equipment management are difficult. Consumable items either goes into recycle after use or it remains inside patients' body such as screws use for fix patients' bone. Although consumable items' management is easier then reusable but sterilisation unit still needs to maintain record for all equipment including consumable items for audit purposes, analysis and research. In case, patients get infection after surgery then the sterilisation unit needs to record those cases in association of equipment used for those patients.

Reusable items management is more difficult due to various functions such as theatre supply, theatre collection, recorded use for each equipment, synchronisation with surgery scheduling, equipment orders with suppliers, equipment washing, packaging, sterilisation and others. Equipment is used in the form of a set of items in surgeries. Every surgery requirement is different and sterilisation needs to fulfil the theatre requirement according to the theatre schedule for every surgery otherwise the surgery cannot perform better. If any patient gets infected then sterilisation needs to back track all the equipment used for particular surgery including individual items within equipment set which may be part of other equipment set after use. In this complex scenario, it is very difficult to keep accurate record. Accuracy of record even more necessary in case of infected equipment handling. RFID can provide automation, minimize the errors and keep track individual items; it allows quick and more efficient information for sterilisation equipment which further enables better equipment management.

\subsection{Notes management}

Patients' notes are important for patients' treatment. Without patients' history and notes doctor cannot see patient. Notes are necessary for in-patients and out-patients. Outpatients such as clinic appointment, if doctor don't have the notes at the time of patients' clinical appointment then appointment needs to be cancel. In this case, hospital resource and time cannot be utilized at optimum level. Staff need in-patients notes on a regular basis in comparison to out-patients notes, due to the frequency of treatment in relation to the following; wards, scan and theatre units. In this case there is a need to see patients' notes at the time of treatment or before treatment. So, it is critical for healthcare practitioners and clerical staff to make patients' notes available at the location and time of treatment. When patients have multiple modalities and multiple staff need to update notes then notes management is even more complex because notes have to move from one place to another very frequently. During transfer process notes can go missing and as well as notes can delay which cause delay or cancellation (depend on medical condition) in treatment. RFID can improve notes management and automate records for notes location which can help staff to visualize the status of the notes. This enables staff to collect notes quickly if necessary without patients' treatment cancellation. It makes the archiving process easy and secure. RFID solution also stops unauthorized access which certainly improves the quality of care. 


\section{RFID connecting model}

It has been investigated in section 9 and 10 that as technology evolves each time, tags and hardware increase their performance for better RFID use. Although it is recommended in figure 14, that the vendor can minimize the complexity at the technological level with consistent technological upgrades. However, there is no single standardization is available at technical level and it is very difficult to achieve standardization at technical level too. Due to lack of standardization it is difficult to rely on one technological solution. In that case, future technological upgrade may affect application (see section 13 \& 14) usability and application may not compatible with new technological upgrades. However, adoption of new advancement in technology is also good for better performance. So, it is better to adapt middle level approach, in which RFID solutions should not stop adaption of new technological advancement and also does not affect application interface. Especially in the case of healthcare application interfaces because healthcare applications their interfaces and integration are really complex. Moreover healthcare applications are significantly big and need major investment. However, it improves overall organisational performance with resource optimization significantly.

This research uses RFID for context management and support practitioners knowledge in real-time environment. Practitioners need constant support with appropriate level of knowledge management interface. Section 14 discusses the various application need to use RFID hardware for constant update of equipment, notes and other stuff within healthcare for better overall healthcare management which is necessary for patient processes. In this connection, a RFID connecting model for healthcare applications is developed, it supports RFID application interface should not affect if RFID solution adapts RFID technology change or upgrade. Figure 17 shows this model, it provide the flexibility to RFID applications to adopt future technology advancements without changing frontend.

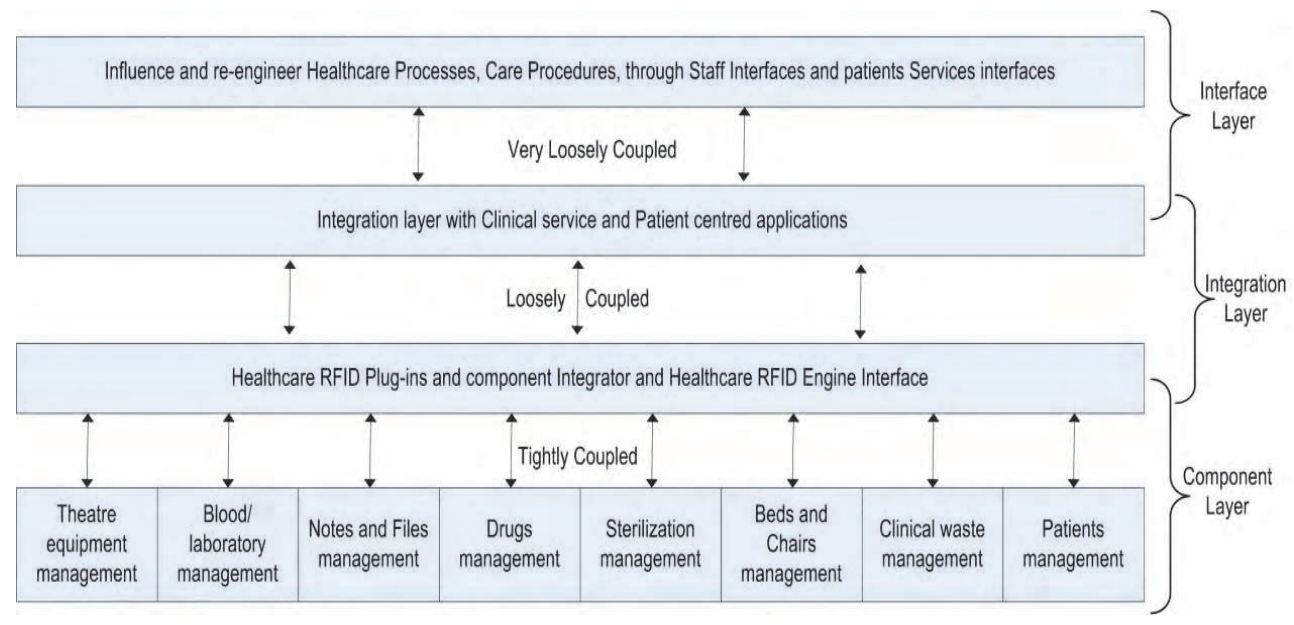

Fig. 17. Hospital RFID application model

It is feasible for staff and healthcare processes to work through the same interface layer. The interface layer should not need changing due to the integration layer which is based on patient centred application and healthcare services, and use RFID engine. The foundation of 
engine interface is based on RFID plug-ins and component integrator. In component layer each management scheme utilizes various types of tags, readers and hardware. Each component such as drugs management, theatre equipment management can use the same or different implementation logic. However, it provides feasibility and flexibility for interaction with healthcare interface through variable set of plug-ins and component integrator (technical procedures). This model further provide the feasibility to integrate all management schemes appropriately for better patients process management which can minimize the error and improvement the performance with resource optimization.

\section{Conclusion}

This chapter considered the RFID components with its potential alternatives and possible healthcare applications. The present research defines and analyses the most important RFID components (tag and reader) with its' alternatives and its use in various situations. It is conceived that RFID is very important for resource optimisation, increasing efficiency within organisational processes, providing enhanced service, and making organisational staff overall experience better. The research observed various cases in healthcare settings and analyses the complexity of healthcare processes. However, it is pragmatic to put RFID for healthcare objects' (e.g. notes, equipments and drugs etc.) tracking for improved healthcare service with optimised use of resources.

The first part of this chapter has explained and described the RFID technology and its components, and the second part has discussed the main considerations of RFID technology in terms of advantages and study model. The last part explores RFID technology applications. This chapter considers RFID technology as a means to provide new capabilities and efficient methods for several applications. For example, in healthcare, access control, analyzing inventory information, and business processes. RFID technology needs to develop its capability to be used with computing devices. This allows businesses to get real potential benefits of RFID technology. This study facilitates adoption of location deduction technology (RFID) in a healthcare environment and shows the importance of the technology in a real scenario and application in connection with resource optimization and improving effectiveness. However, there is no doubt in the future that many companies and organisations will benefit from RFID technology especially healthcare.

\section{Acknowledgment}

We would like to thank the hospital management and NHS Trust chair for allowing us access to the hospital for our research. We are grateful to all the hospital staff: managers, surgeons, doctors, IT managers, IT developers, nurses and ward staff for their support and time in providing us with information about patients' movements for medical treatment within the hospital. The resulting knowledge and analysis has provided a useful foundation for our research in exploiting the RFID usability for healthcare.

\section{References}

Application Notes CAENRFID, (2008), Introduction to RFID Technology, CAENRFID: The Art of Identification 
Bharadwaj, V., Raman, R., Reddy, R. \& Reddy, S., (2001), Empowering mobile healthcare providers via a patient benefits authorization service, WET ICE 2001. Proceedings. Tenth IEEE International Workshops on Enabling Technologies: Infrastructure for Collaborative Enterprises, IEEE.

Bohn, J., (2008), Prototypical implementation of location-aware services based on a middleware architecture for super-distributed RFID tag infrastructures, Personal Ubiquitous Computing, ACM, 12 (2):155-166.

Connecting for health, http://www.connectingforhealth.nhs.uk/systemsandservices /nhsmail/using [access: 11th October, 2009].

Connecting for health, http://www.connectingforhealth.nhs.uk/ [access: 18th August, 2010].

DHS (Department of Homeland Security) , (2006), Enhanced Security Controls needed for US-Visit's System using RFID Technology, U.S. Department of Homeland Security (Office of Inspector General), available at: www.dhs.gov/xoig/assets/mgmtrpts /OIG_06-39_Jun06.pdf, OIG-06-39.

DH-UK, http://www.dh.gov.uk/en/index.htm [access: 30th September, 2009].

Frank, T., Brad, H., Anand, M., Hersh, B., Anita, C. \& John, K., (2006), RFID Security, ISBN: 1-59749-047-4.

GAO (Government Accountability Office), (2005), Information Security: Radio Frequency Identification Technology in the Federal Government, Report to Congressional Requesters, US. Government Accountability Office, available at: www.gao.gov/new.items/d05551.pdf, GAO-05-551.

Garfinkel, S. \& Rosenberg, B., (2005), RFID Application, Security, and Privacy, ISBN: 0-32129096-8.

Glover, B. \& Bhatt, H., (2006) RFID Essentials, O'Reilly Media, Inc, Sebastopol, ISBN 0-59600944-5.

Intermec, (2009), ABCs of RFID: Understanding and using radio frequency identification, White Paper, Intermec Technologies Corporation, available at:

http://epsfiles.intermec. com/eps_files/eps_wp/ABCsofRFID_wp_web.pdf [access: 3rd January, 2010].

Meiller, Y. \& Bureau, S. (2009), Logistics Projects: How to Assess the Right System? The Case of RFID Solutions in Healthcare, Americas Conference on Information Systems (AMCIS) 2009 Proceedings, Association for Information Systems.

Narayanan, A., Singh, S. \& Somasekharan, M., (2005), Implementing RFID in Library: Methodologies, Advantages and Disadvantages, Scientific Information Resource Division, IGCAR, Kalpakkam, Government of India, available at: http://library.igcar.gov. in/readit-2005/conpro/lgw/s5-8.pdf [access: 15th February, 2010].

NHS-UK, http:/ / www.nhs.uk/Pages/HomePage.as px [access: 11th October, 2009].

Parks, R., Yao, W. \& Chu, C. H., (2009), RFID Privacy Concerns: A Conceptual Analysis in the Healthcare Sector, Americas Conference on Information Systems (AMCIS) 2009 Proceedings, Association for Information Systems.

Sandip, L., (2005), RFID Sourcebook, IBM Press, ISBN: 0-13-185137-3.

Schwieren1, J. \& Vossen, G., (2009), A Design and Development Methodology for Mobile RFID Applications based on the ID-Services Middleware Architecture, Tenth 
International Conference on Mobile Data Management: Systems, Service and Middleware, IEEE Computer Society.

Shepard, S., (2005), RFID Radio Frequency Identification, McGraw-Hill, ISBN:0-07-144299-5.

Srivastava, L., (2005), RFID: Technology, Applications and Policy Implications, Spectrum Management Workshop, International Telecommunication Union, available at: http://www.itu.int/osg/spu/presentations/2005/srivastavaRFID2005.pdf.

Watson, M., (2006), Mobile healthcare applications: a study of access control, Proceedings of the 2006 International Conference on Privacy, Security and Trust: Bridge the Gap Between PST Technologies and Business Services, ACM, article no. 77, DOI: http://doi.acm.org/10.1145/1501434.1501524.

Zeisel, E. \& Sabella, R. (2006), RFID+ Exam Cram, Pearson, Series 2, ISBN: 0-7897-3504-0. 


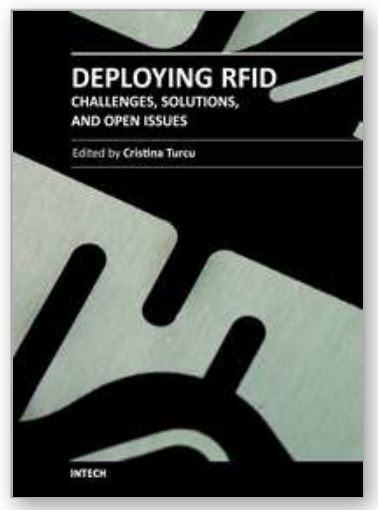

\author{
Deploying RFID - Challenges, Solutions, and Open Issues \\ Edited by Dr. Cristina Turcu
}

ISBN 978-953-307-380-4

Hard cover, 382 pages

Publisher InTech

Published online 17, August, 2011

Published in print edition August, 2011

Radio frequency identification (RFID) is a technology that is rapidly gaining popularity due to its several benefits in a wide area of applications like inventory tracking, supply chain management, automated manufacturing, healthcare, etc. The benefits of implementing RFID technologies can be seen in terms of efficiency (increased speed in production, reduced shrinkage, lower error rates, improved asset tracking etc.) or effectiveness (services that companies provide to the customers). Leading to considerable operational and strategic benefits, RFID technology continues to bring new levels of intelligence and information, strengthening the experience of all participants in this research domain, and serving as a valuable authentication technology. We hope this book will be useful for engineers, researchers and industry personnel, and provide them with some new ideas to address current and future issues they might be facing.

\title{
How to reference
}

In order to correctly reference this scholarly work, feel free to copy and paste the following:

Kamran Ahsan (2011). RFID Components, Applications and System Integration with Healthcare Perspective, Deploying RFID - Challenges, Solutions, and Open Issues, Dr. Cristina Turcu (Ed.), ISBN: 978-953-307-380-4, InTech, Available from: http://www.intechopen.com/books/deploying-rfid-challenges-solutions-and-openissues/rfid-components-applications-and-system-integration-with-healthcare-perspective

\section{INTECH}

open science | open minds

\author{
InTech Europe \\ University Campus STeP Ri \\ Slavka Krautzeka 83/A \\ 51000 Rijeka, Croatia \\ Phone: +385 (51) 770447 \\ Fax: +385 (51) 686166 \\ www.intechopen.com
}

\author{
InTech China \\ Unit 405, Office Block, Hotel Equatorial Shanghai \\ No.65, Yan An Road (West), Shanghai, 200040, China \\ 中国上海市延安西路65号上海国际贵都大饭店办公楼405单元 \\ Phone: +86-21-62489820 \\ Fax: $+86-21-62489821$
}


(C) 2011 The Author(s). Licensee IntechOpen. This chapter is distributed under the terms of the Creative Commons Attribution-NonCommercialShareAlike-3.0 License, which permits use, distribution and reproduction for non-commercial purposes, provided the original is properly cited and derivative works building on this content are distributed under the same license. 